\title{
Inhibition of Rho kinase protects against colitis in mice by attenuating intestinal epithelial barrier dysfunction via MLC and the NF-кB pathway
}

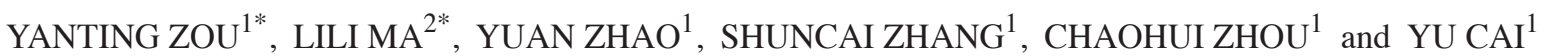 \\ ${ }^{1}$ Department of Gastroenterology and Hepatology, ${ }^{2}$ Endoscopy Center, Zhongshan Hospital, \\ Fudan University, Xuhui, Shanghai 200032, P.R. China
}

Received June 14, 2017; Accepted October 9, 2017

DOI: $10.3892 /$ ijmm.2017.3197

\begin{abstract}
The aim of the present study was to investigate the role of Rho kinase (also known as ROCK) inhibitor in 2,4,6-trinitrobenzene sulfonic acid induced mouse colitis; and to elucidate the underlying mechanism of ROCK1/ROCK2 inhibition in enhancing intestinal epithelial barrier (IEB) function. A specific inhibitor of ROCK, Y-27632, was used to examine the role of ROCK in mouse colitis models. ROCK1 and ROCK2 were silenced respectively using RNA interference in Caco- 2 cells. The expression of tight junction proteins and the downstream molecules of ROCK were assessed. Y-27632 alleviated colonic inflammation and decreased intestinal permeability. ROCK-myosin light chain (MLC) and ROCK-NF- $\kappa \mathrm{B}$ pathway were activated in colitis and inhibited by Y-27632. In vitro, ROCK1 RNAi primarily downregulated the phosphorylation of myosin phosphatase-targeting subunit-1 (MYPT-1) and MLC, while ROCK2 RNAi inhibited phosphorylation of nuclear factor $-\kappa \mathrm{B}(\mathrm{NF}-\kappa \mathrm{B})$. In conclusion, the results suggested that the ROCK inhibitor alleviated colitis and IEB dysfunction. Inhibition of phospho-MYPT-1 and MLC by ROCK1 knockout or inhibition of NF- $\mathrm{B}$ phosphorylation by ROCK2 knockout may be the underlying mechanisms.
\end{abstract}

\section{Introduction}

Inflammatory bowel disease (IBD) is one of the chronic nonspecific inflammatory diseases of the intestine, characterized by a leaky intestinal epithelial barrier (IEB). Breakdown of the IEB is observed in both IBD models and patients $(1,2)$.

Correspondence to: Dr Yu Cai or Dr Chaohui Zhou, Department of Gastroenterology and Hepatology, Zhongshan Hospital, Fudan University, 180 Fenglin Road, Xuhui, Shanghai 200032, P.R. China

E-mail: cai.yu@zs-hospital.sh.cn

E-mail: zhou.chaohui@zs-hospital.sh.cn

*Contributed equally

Key words: Rho kinase, intestinal epithelial barrier, tight junction, myosin light chain, nuclear factor- $\kappa \mathrm{B}$
IEB dysfunction is also found in some healthy relatives of IBD patients, which suggests that IEB dysfunction is not just the result of IBD, it may be the reason (3-5).

The tight junction proteins (TJs) are important components of IEB, forming seals between adjacent epithelial cells near the apical surface, thus preventing paracellular diffusion of microorganisms and other antigens across the epithelium. Most scientists believe that the cytoskeletal structure alteration followed by redistribution of TJs leading to breakdown of the IEB $(6,7)$. TJs are composed of multiple proteins including transmembrane proteins (such as occludin, tricellulin, claudins and junctional adhesion molecule) and intracellular proteins [such as zonula occludens (ZO)-1, -2, -3 and cingulin] (8). It has been reported that tumor necrosis factor- $\alpha$ (TNF- $\alpha)$, nuclear factor- $\kappa \mathrm{B}(\mathrm{NF}-\kappa \mathrm{B})$ and myosin light chain kinase signaling pathway could regulate $\mathrm{TJ}$ function $(9,10)$.

Rho kinase (also known as ROCK) is an important regulator of cytoskeleton, which was originally identified as an effector of small GTPase Rho (11). Two isoforms of ROCK have been identified in mammalian system: ROCK1 (also known as ROK $\beta$ or p160 ROCK) and ROCK2 (also known as ROK $\alpha$ ). ROCK1 and ROCK2 share an overall 65\% homology at amino-acid level and 92\% homology in kinase domains (12). Despite their similar kinase domain, ROCK1 and ROCK2 still serve different functions. ROCK1 is specifically cleaved by caspase-3 $(13,14)$, whereas ROCK2 is cleaved by granzyme B or caspase-2 $(15,16)$. ROCK1 is essential for the formation of stress fibers (17), whereas ROCK2 appears to be necessary for phagocytosis and cell contraction (18). Until this point, the differences of these two isoforms in the recognition of downstream targets remain unclear.

Several studies have demonstrated that ROCK participated in the process of intestinal inflammation and epithelial barrier dysfunction. Ivanov et al (19) and Segain et al (20) reported RhoA activated in inflamed intestinal mucosa in experimental colitis rats and in Crohn's disease patients. Mihaescu et al (21) also found that Y-27632 (one of the ROCK inhibitors) could relieve the IEB damage in radiation-induced intestinal inflammation.

There are varies of downstream factors of ROCK, such as myosin phosphatase-targeting subunit-1 (MYPT-1) of myosin light chain phosphatase (MLCP) and MLC. ROCK may 
activate MLC directly or in an indirect pattern by activating MYPT-1. Since MYPT-1 is a negative regulator of MLCP, activating of MYPT-1 may reduce the de-phosphorylation of MLC by MLCP, which finally lead to the activation of MLC $(11,12)$. Another downstream pathway of ROCK is the $\mathrm{NF}-\kappa \mathrm{B}$ pathway $(22,23)$. After ROCK activation, I $\mathrm{B} \alpha$ is phosphorylated and rapidly degraded, allowing $N F-\kappa B$ to transmigrate within the nucleus and induce inflammatory cytokine gene transcription by binding to specific promoter elements.

However, the precise molecular mechanisms of action of ROCK in the intestinal inflammation and IEB dysfunction remain unknown. In the present study, the authors used Y-27632 to evaluate the role of ROCK inhibition in the intestinal inflammation and barrier dysfunction in a 2,4,6-trinitrobenzene sulfonic acid (TNBS)-induced colitis mouse model. The downstream molecules of ROCK were also examined to clarify the alteration of the ROCK signaling pathway during the experimental colitis. In order to evaluate the differences of ROCK1 and ROCK2 in recognizing downstream molecules, the authors used RNA interference technique to silence ROCK1 and ROCK2 separately in human colon cancer cell lines (Caco-2). The results suggested a significant role of ROCK inhibitor in intestinal inflammation and barrier dysfunction. Furthermore, the results suggested ROCK1 mainly modulates phosphorylation of MYPT-1 and MLC, while ROCK2 regulates phosphorylation of $N F-\kappa B$.

\section{Materials and methods}

Induction of mouse colitis. Animal experiments were performed in accordance with the guidelines on the protection of animals and were approved by the Ethics Committee for Animal Experimentation of Fudan University (Shanghai, China). A total of 60 male BALB/c mice, 4-5 weeks old and weighing 18-22 g, were obtained from the Animal Center of Fudan University (Shanghai, China), maintained in an environmentally controlled room $\left(23 \pm 2^{\circ} \mathrm{C}, 55 \pm 10 \%\right.$ humidity) with a $12 \mathrm{~h} \mathrm{light/dark} \mathrm{cycle} \mathrm{and} \mathrm{free} \mathrm{access} \mathrm{to} \mathrm{food} \mathrm{and}$ water. Colitis was induced by intra-colonic administration of TNBS solution $(150 \mathrm{mg} / \mathrm{kg}$ in $50 \%$ ethanol) once daily for up to 7 days. Y-27632, dissolved in saline, was administered by intraperitoneal injection at a dose of $10 \mathrm{mg} / \mathrm{kg}$ body weight just following enteroclysis on a daily basis.

A total of 60 mice were randomly divided into 3 groups as follows: group $1(\mathrm{n}=10)$ receiving saline through intracolonic and intraperitoneal injection; group $2(n=30)$ receiving intracolonic administration of TNBS/ethanol solution and intraperitoneal injection of saline; group $3(n=20)$ receiving intracolonic administration of TNBS/ethanol solution and intraperitoneal injection of Y-27632.

Diarrhea and bloody stool extents were recorded every day, calculated according to Mayo Score as previously described with modification (24). The final diarrhea score of each group was expressed as: 1 , normal; 2, 1-2 stools/day more than normal; 3, 3-4 stools/day more than normal; 4, >4 stools/day more than normal. The bloody stool score was recorded as: 1 , none; 2 , visible blood with stool less than half the time; 3 , visible blood with stool half of the time or more; 4 , passing blood alone.
Evaluation of colonic inflammation. The proximal $\sim 1.0 \mathrm{~cm}$ of the colonic segment was used for histological examination. The segment was fixed in $4 \%$ formaldehyde and embedded in paraffin. A morphometric analysis was performed in a blinded fashion by two investigators on haematoxylin and eosin stained $4 \mu \mathrm{m}$ transverse sections. The extent of colonic inflammation was assessed using the scoring system described by Ameho et al (25).

Measurement of intestinal permeability. Mice (10 from each group) were anesthetized by an intraperitoneal injection of $3.5 \%$ chloral hydrate $(10 \mathrm{ml} / \mathrm{kg}$ body weight $)$ on day 7 before being sacrificed by cervical dislocation. A $10 \mathrm{~cm}$ distance of terminal ileum ligation was performed at $\sim 5 \mathrm{~cm}$ proximal to the ileocecal valve. Then a solution containing $20 \mathrm{mg} / \mathrm{ml}$ fluorescein isothiocyanate (FITC)-conjugated dextran (FITC-dextran, molecular weight $4.4 \mathrm{kDa}$; Sigma-Aldrich; Merck KGaA, Darmstadt, Germany) in a total volume of $0.5 \mathrm{ml}$ phosphate-buffered saline (PBS) was injected into the ligatured ileum. At $30 \mathrm{~min}$ following injection, blood samples $(1 \mathrm{ml})$ were obtained from the peripheral vessel and centrifuged $\left(3,000 \mathrm{x} \mathrm{g}\right.$ at $\left.4^{\circ} \mathrm{C}\right)$ for $1 \mathrm{~min}$; then $100 \mu \mathrm{l}$ plasma was mixed with $0.9 \mathrm{ml} \mathrm{PBS}$ and added to a 96 -well microplate. The concentration of FITC-dextran was then determined by spectrophotofluorometry with an excitation wavelength of $480 \mathrm{~nm}$ and an emission wavelength of $520 \mathrm{~nm}$.

Transmission electron microscopy (TEM). Glutaraldehydefixed specimens were washed in PBS and immersed in $1 \% \mathrm{OsO}_{4}$. Following dehydration in graded ethanol and immersion in the intermedium toluol, the specimens were embedded in epoxy resin (Serva Electrophoresis $\mathrm{GmbH}$, Heidelberg, Germany). Semithin $(500 \mathrm{~nm})$ and thin $(50 \mathrm{~nm})$ sections were cut with an ultramicrotome. Semithin sections were stained with $1 \%$ alkalinized toluidine blue, then cut into thin sections. Thin sections were stained with uranyl acetate and lead citrate, and examined by electron microscope Zeiss EM 10 CR (Zeiss AG, Oberkochen, Germany).

Immunohistochemistry. Sections (4 $\mu \mathrm{m})$ of formalin fixed paraffin embedded tissues were mounted on probe-on slides, deparaffinized in xylene, and rehydrated in distilled $\mathrm{H}_{2} \mathrm{O}$ through graded alcohol. Sections were blocked with normal mouse serum for $30 \mathrm{~min}$ and incubated overnight at $4^{\circ} \mathrm{C}$ with occludin antibody $(1: 150 ; 13409-1-\mathrm{AP})$ and ZO-1 antibody (1:150; 21773-1-AP) (both from ProteinTech Group, Inc., Chicago, IL, USA). The sections were subsequently washed and incubated with SignalStain Boost HRP-Polymer solution (8114; Cell Signaling Technology, Danvers, MA, USA) for $30 \mathrm{~min}$ at room temperature, followed by incubation for 5-10 min with 3,3'-diaminobenzidine tetrachloride (D8001; Sigma-Aldrich, St. Louis, MO, USA). Cells were considered as positive once the dark-yellow granules were present either on the membrane or in the cytoplasm. Five randomized microscopic views of 400-fold magnification of each section were observed in a blinded fashion by one pathologist and a semiquantitative scoring system was based on both the staining intensity ( 0 , negative; 1 , weak; 2 , intermediate; 3 , strong) and the percentage of positive cells $(0,0 \%$ positive cells; $1,1-10 \%$ positive cells; $2,11-50 \%$ positive cells; $3,>50 \%$ positive cells). 
The final score of each sample was obtained by multiplying the scores of staining intensity and percentage of positive cells.

Cell culture. Caco-2 cells were purchased from the Shanghai Cell Bank of Chinese Academy of Science (Shanghai, China) and cultured in RPMI-1640 medium containing $15 \%$ fetal bovine serum. Cells were seeded at $1 \times 10^{6}$ cells/well in 6-well plates and grown to confluence. Caco-2 cells were then treated with either vehicle (PBS) or $10 \mathrm{ng} / \mathrm{ml}$ recombinant human TNF- $\alpha$ (Sigma-Aldrich) for $24 \mathrm{~h}$.

Small interfering RNA (siRNA) transfection. The authors chose lentivirus pGLV-H1-GFP+Puro (LV3) as a shuttle vector. The siRNAs targeting human ROCK1 and ROCK2 were as follows: ROCK1, GCATTTGGAGAAGTTCAATTG, GCAGAAGTAG TTCTTGCATTG, GCACCAGTTGTACCCGATTTA and GGA TGAAGAGGGAAATCAAAG; ROCK2, GGTTTATGCTAT GAAGCTTCT, GGAAGAAATCAGACAGCATCC, GCAGA ACAGTATTTCTCAACC and GCTTCTCTTGAGGAAACT AAT. The siRNAs and nonspecific control siRNA duplexes were synthesized, desalted and purified by Shanghai GenePharma Co.,

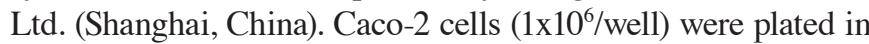
6-well plates and were cultured to confluence in RPMI-1640 medium. siRNA, pLV/helper-SL3, pLV/helper-SL4 and pLV/helper-SL5 (4 $\mu \mathrm{g}$ each) and $40 \mu \mathrm{l}$ Lipofectamine 2000 were mixed with $3 \mathrm{ml}$ serum free Opti-MEM I solution to form a transfection mixture. Following siRNA transfection for $48 \mathrm{~h}$, cells were treated with either vehicle (PBS) or $10 \mathrm{ng} / \mathrm{ml}$ TNF- $\alpha$ for another $24 \mathrm{~h}$. The knockdown efficiency was assessed by reverse transcription-quantitative polymerase chain reaction (RT-qPCR) and western blotting after $72 \mathrm{~h}$.

$R T-q P C R$. Total RNA of the colon and Caco-2 cell were isolated using TRIzol reagent (Takara Bio, Inc., Ostu, Japan) according to the manufacture's protocol. The primers were as follows: occludin (mouse): (F) 5'-TTCAAACCCAATCATTATGC ACC-3' and (R) 5'-AAGAGTACGCTGGCTGAGAGAGC-3'; ZO-1 (mouse): (F) 5'-TTCCAGAACCGAAACCTGTGT ATG-3' and (R) 5'-GGCAGAGCACCATCAGAAGGG-3'; ROCK1 (mouse and human): (F) 5'-TGATTCTGAGATGATTG GAGACCTTC-3' and (R) 5'-GAGTGATTAAGCATGTCTTG AGCCTC-3'; ROCK2 (mouse and human): (F) 5'-GAGACA ACTGGATGAAACCAATGC-3' and (R) 5'-GAATCTGTT TTGAACTTTCTGCCTG-3'; glyceraldehyde 3-phosphate dehydrogenase (GAPDH) (mouse): (F) 5'-CATGAGAAGTAT GACAACAGCCT-3' and (R) 5'-AGTCCTTCCACGATA CCAAAGT-3'; occludin (human): (F) 5'-AGTGAATGACAA GCGGTTTTATCC-3' and (R) 5'-CACAGGCGAAGTTAA TGGAAGC-3'; ZO-1 (human): (F) 5'-GAGCACAGCAAT GGAGGAAACAG-3' and (R) 5'-AAATGAGGATTATCT CGTCCACCAG-3'; $\beta$-actin (human): (F) 5'-AGTGTGACG TTGACATCCGTA-3' and (R) 5'-GCCAGAGCAGTAATC TCCTTCT-3'. The First Strand cDNA of each sample was synthesized from $2 \mu \mathrm{g}$ total RNA using SuperScript II according to the manufacturer's instruction (Invitrogen; Thermo Fisher Scientific, Inc., Waltham, MA, USA). The reactions were as follows: Pre-denaturation at $95^{\circ} \mathrm{C}$ for $3 \mathrm{~min}$ followed by 40 cycles of $95^{\circ} \mathrm{C}$ for $12 \mathrm{sec}$ (denaturation) and $62^{\circ} \mathrm{C}$ for $40 \mathrm{sec}$ (annealing/extension). The fluorescent products were detected by LightCycle system (BioRad IQ5;
Bio-Rad Laboratories, Inc., Hercules, CA, USA) before the completion of each cycle.

Western blot analysis. Both colon samples and Caco-2 cells were lysed and homogenized in ice-cold radioimmunoprecipitation assay lysis buffer (Beyotime Institute of Biotechnology, Haimen, China). Proteins were loaded onto each well of sodium dodecyl sulfate-polyacrylamide gel electrophoresis (SDS-PAGE) ready gels (Bio-Rad Laboratories, Inc.) for electrophoresis. Proteins were transferred onto a nitrocellulose membrane (Bio-Rad Laboratories, Inc.) by electro blotting. The membrane was washed and blocked with $5 \%$ milk in TBS with $0.05 \%$ Tween-20, and incubated overnight with specific primary antibodies at $4^{\circ} \mathrm{C}$. Occludin antibody (1:500; 13409-1-AP), ZO-1 antibody (1:500; 21773-1-AP), GAPDH antibody $(1: 2,000 ; 10494-1-A P), \beta$-actin antibody $(1: 1,000$; 20536-1-AP) (all from ProteinTech Group, Inc.), NF-кB p65 rabbit $\mathrm{mAb}(1: 1,000 ; 8242)$, phospho-NF- $\mathrm{kB}$ p65 (Ser536) antibody $(1: 1,000 ; 3031)$ (both from Cell Signaling Technology, Inc.), ROCK1 antibody (1:200; 21850-1-AP), ROCK2-Specific (C-Term) antibody (1:200; 20248-1-AP) (both from ProteinTech Group, Inc.), phospho-MYPT-1 (Thr696) antibody (1:1,000; 5163) and phospho-Myosin Light Chain 2 (Ser19) antibody $(1: 1,000 ; 3671)$ (both from Cell Signaling Technology, Inc.) were used as the primary antibodies. Goat anti-rabbit IgG-HRP (1:1,000; SC-2004; Santa Cruz Biotechnology, Inc., Santa Cruz, CA, USA) was incubated as the secondary antibody at room temperature for $1 \mathrm{~h}$. Membranes were washed and the assessed proteins were detected using an enhanced chemiluminescence reagent (GE Healthcare Life Sciences, Chalfont, UK). Relative intensity of the bands was quantified using Gel-Pro Analyzer 6.3 (Media Cybernetics LP, Silver Spring, MD, USA).

Statistical analysis. Results were expressed as mean \pm standard error of mean. Data expressed as percentages were analyzed using Chi-square test. Data with normal distribution were compared by one-way analysis of variance and Student's t-test. Mann-Whitney U test was performed in non-normal distribution data. Log-rank (Mantel-Cox) test was used for survival curve. The statistical analysis was performed using GraphPad Prism 5 (GraphPad Software, Inc., La Jolla, CA, USA). $\mathrm{P}<0.05$ was considered to indicate a statistically significant difference.

\section{Results}

ROCK inhibitor attenuates TNBS-induced colitis in mice. By the end of TNBS administration, 14 mice died in group 2 $(14 / 30,46.7 \%)$ and 5 died in group $3(5 / 20,25 \%)$, respectively. All mice remained alive in group 1 . The survival curve was analyzed using log-rank (Mantel-Cox) test $(\mathrm{P}<0.05)($ Fig. 1A). From day 3 , mice in group 2 began to present significant diarrhea, bloody stool, weight loss and inactivity; while mice in group 3 presented slight diarrhea and less bloody stool; no diarrhea and bloody stool was found in group 1 (Fig. 1B and C).

Moreover, the authors found that Y-27632 treatment significantly ameliorated the colonic inflammation. In group 2 , inflammatory infiltration was observed throughout the whole colonic wall, apparent folliculus lymphatics hyperplasia in the lamina propria, some hemorrhagic focus in the submucosa 

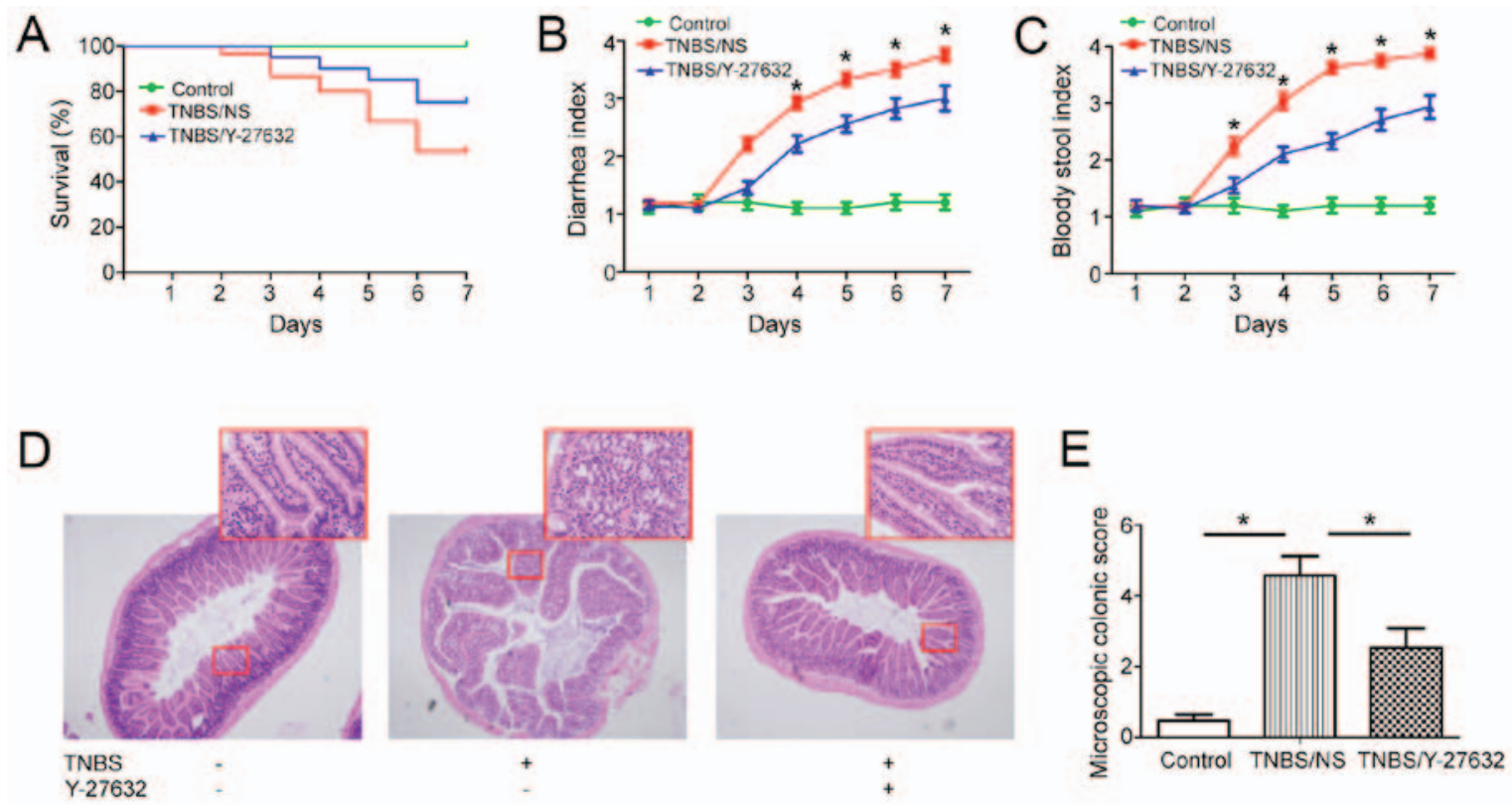

Figure 1. Diarrhea and bloody stool improved and colonic inflammation ameliorated by Y-27632 in TNBS-induced colitis mice. (A) Survival curve of three groups, and data analyzed by log-rank (Mantel-Cox) test ( $\mathrm{P}=0.0275)$. (B) Effect of Y-27632 on mouse diarrhea in 7 days. (C) Effect of Y-27632 on mouse bloody stool in 7 days prior to sacrifice. (D) Typical patterns of hematoxylin and eosin staining for colonic inflammation evaluation of mice after TNBS and/or Y-27632 treatment for 7 days (magnification, $\mathrm{x} 40$ and $\mathrm{x} 400$ ). (E) Colitis Ameho score of three groups. Values are expressed as mean \pm standard error of the mean. ${ }^{~} \mathrm{P}<0.05$ for TNBS/Y-27632 vs. TNBS/NS and for TNBS/NS vs. control. Control group, $\mathrm{n}=10$; TNBS/NS group, $\mathrm{n}=16 ; \mathrm{TNBS} / \mathrm{Y}-27632$ group, $\mathrm{n}=15$. TNBS, 2,4,6-trinitrobenzene sulfonic acid. NS, normal saline.
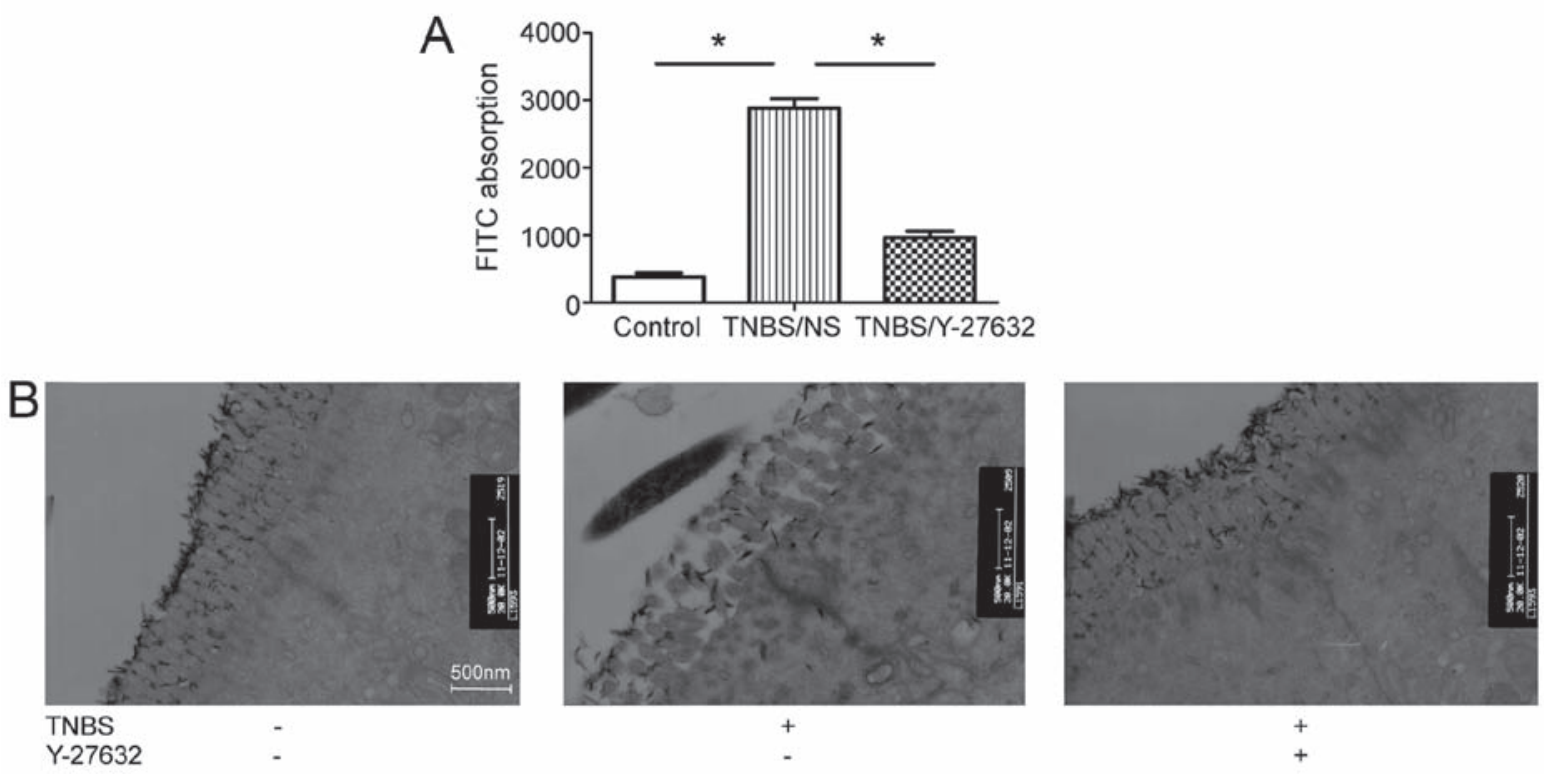

Figure 2. Decreased intestinal permeability and enhanced intestinal epithelial barrier in TNBS-induced colitis mice by Y-27632. (A) Effect of Y-27632 on the levels of FITC-dextran passing from the bowel lumen into the blood circulation in TNBS induced colitis mice. "P $<0.05$ as indicated. Control group, $\mathrm{n}=10$; TNBS/NS group, $n=16$; TNBS/Y-27632 group, $n=15$. (B) Typical patterns of TJs morphology through TEM of mice after TNBS and/or Y-27632 treatment for 7 days. Values are expressed as means \pm standard error of the mean. TNBS, 2,4,6-trinitrobenzene sulfonic acid. NS, normal saline; TJs, tight junction proteins.

when compared with those in group 1, and they obviously alleviated in group 3 (Fig. 1D and E).

ROCK inhibitor regulates intestinal permeability and IEB in TNBS-induced colitis in mice. Intestinal permeability was evaluated by quantifying the levels of FITC-dextran passing from the bowel lumen into the blood circulation. Obviously, TNBS induced increased intestinal permeability by $\sim 8$-fold compared with group 1 and inhibition of ROCK decreased the intestinal permeability (Fig. 2A). The authors observed the morphology of TJs, the primary components of IEB, through TEM. As presented in Fig. 2B, TNBS treatment caused colonic epithelial villus damage, intercellular gap slightly widen, and tracer extravasation when compared to group 1. Y-27632 injection partially protected colonic epithelial villus shape, decrease intercellular gap width and tracer extravasation in group 3. 

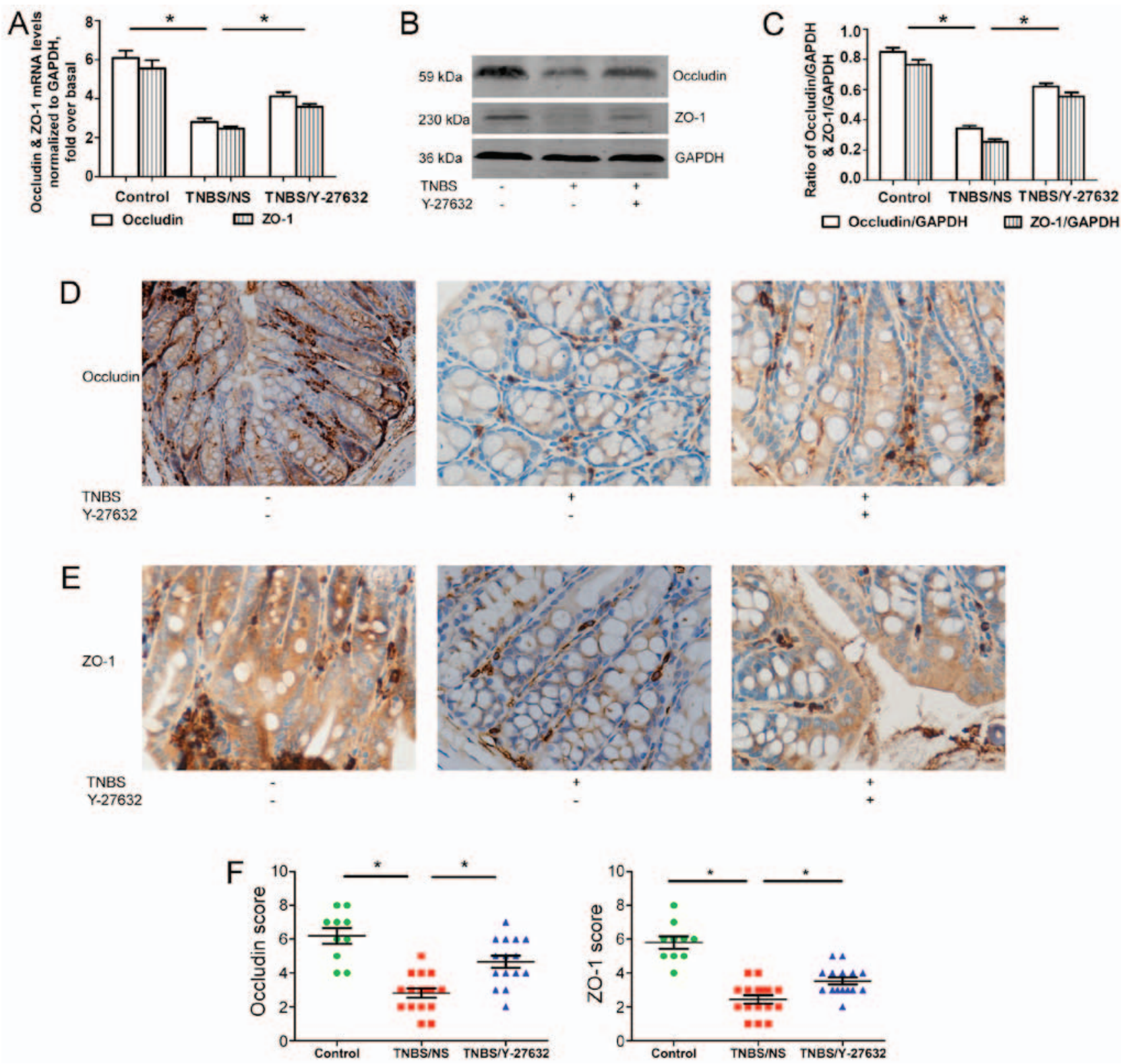

Figure 3. Increased occludin and ZO-1 in TNBS-induced colitis mice by Y-27632. (A) The levels of occludin and ZO-1 mRNA expression determined by reverse transcription-quantitative polymerase chain reaction. Occludin and ZO-1 levels were normalized with GAPDH expression in each sample. (B) Western blotting for occludin and ZO-1 protein levels shown as representative photos. (C) Bars representing the relative protein quantification of occludin and ZO-1 on the basis of GAPDH. (D) Typical patterns of occludin staining in Y-27632 and TNBS-treated mouse colon (magnification, x200). (E) Typical patterns of ZO-1 staining in Y-27632 and TNBS-treated mouse colon (magnification, x200). (F) Scores of immunohistochemistry staining of occludin and ZO-1 in three groups of mouse colon. Values are expressed as means \pm standard error of the mean. " $\mathrm{P}<0.05$ as indicated. Control group, $\mathrm{n}=10, \mathrm{TNBS} / \mathrm{NS}$ group, $\mathrm{n}=16$; TNBS/Y-27632 group, n=15. ZO-1, zonula occludens-1; TNBS, 2,4,6-trinitrobenzene sulfonic acid. NS, normal saline; GAPDH, glyceraldehyde 3-phosphate dehydrogenase.

Furthermore, two main TJs, occludin and ZO-1, were detected separately at mRNA and protein levels using RT-qPCR (Fig. 3A), western blotting (Fig. 3B and C) and immunohistochemistry (Fig. 3D-F). The results showed that the expressions of both occludin and $\mathrm{ZO}-1$ were reduced apparently in group 2 compared with group 1. Y-27632 treatment may elevate occludin and ZO-1 levels.

ROCK1/ROCK2 and their downstream molecules are inhibited by Y-27632 in mice with colitis. ROCK1 and ROCK2 mRNA and protein levels increased in group 2 compared to group 1; while ROCK inhibitor obviously downregulated both
ROCK1 and ROCK2 levels in group 3 (Fig. 4A-C). Then, MYPT-1 and MLC, as the main downstream molecules of the ROCK signaling pathway were tested using western blotting. As shown in Fig. 4B and D, there was a significant increase of phosphorylation of MLC in group 2 compared to that in group 1; while Y-27632 inhibited the phosphorylation of MLC in group 3. The phosphorylated MYPT-1 was similar in group 2 compared with group 1; still Y-27632 treatment in group 3 inhibited the phosphorylation of MYPT-1. In addition, the authors measured the activation of the NF- $\mathrm{KB}$ pathway by detecting the expression of total NF- $\kappa \mathrm{B}$ p65 and phosphorylated NF-kB p65 (Ser536) using western blotting. 

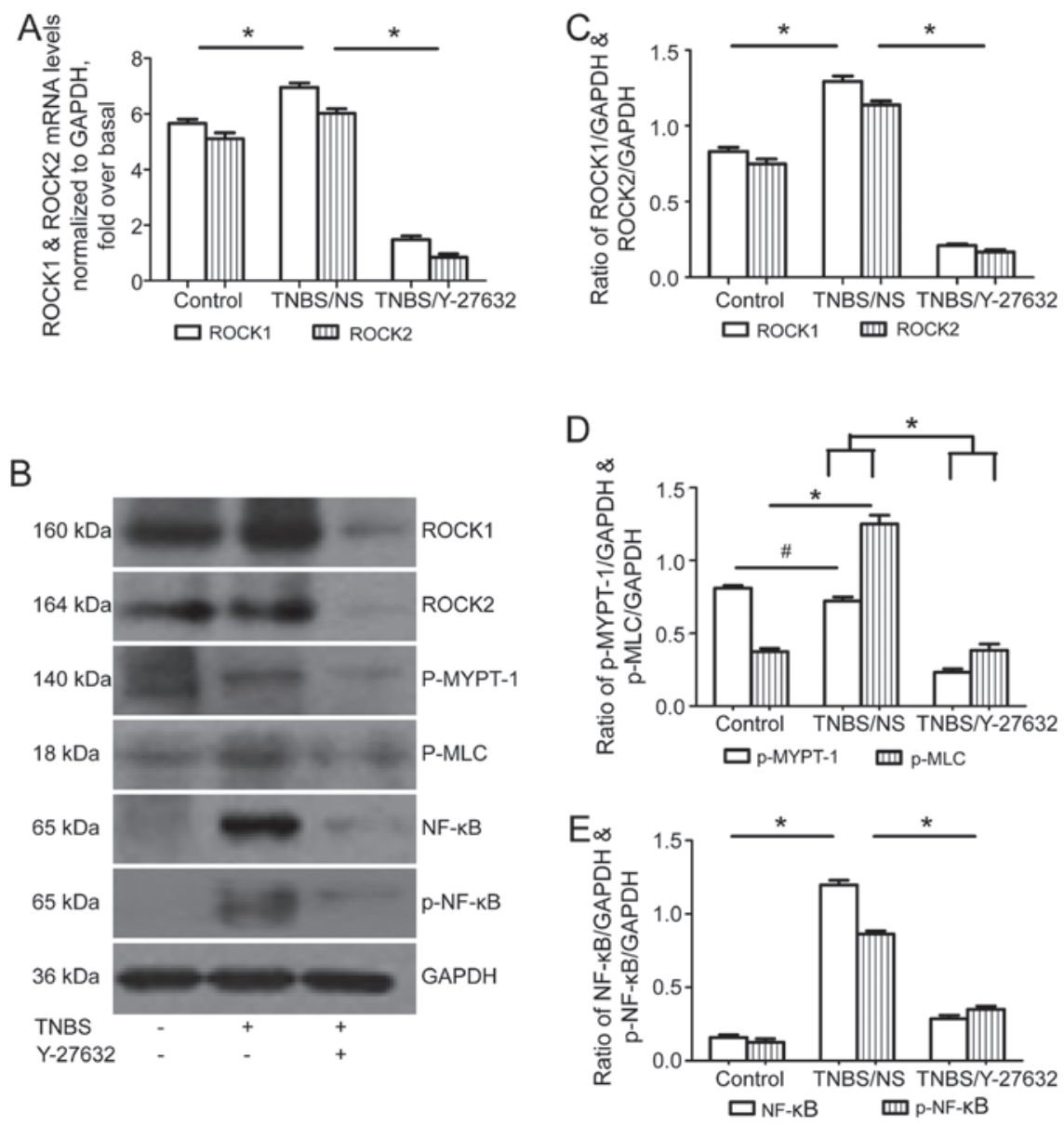

Figure 4. Effect of Y-27632 on ROCK expression levels and its downstream signaling pathway in TNBS-induced colitis mice. (A) The levels of ROCK1 and ROCK 2 mRNA expression determined by reverse transcription-quantitative polymerase chain reaction. ROCK1 and ROCK2 mRNA levels were normalized with GAPDH expression in each sample. " $\mathrm{P}<0.05$ as indicated. (B) Western blotting for ROCK1, ROCK2, p-MYPT-1, p-MLC, NF- $\mathrm{B}$ p65 and p-NF-kB p65 protein levels shown as representative images. (C) Bars representing the relative protein quantification of ROCK1 and ROCK2 on the basis of GAPDH. (D) Bars representing the relative protein quantification of p-MYPT-1 and p-MLC on the basis of GAPDH. (E) Bars representing the relative protein quantification of

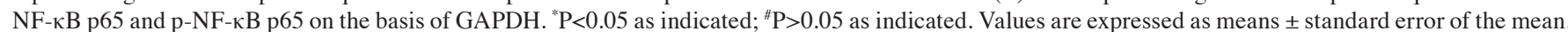
Control group, $\mathrm{n}=10$; TNBS/NS group, $\mathrm{n}=16$; TNBS/Y-27632 group, $\mathrm{n}=15$. ROCK, Rho kinase; TNBS, 2,4,6-trinitrobenzene sulfonic acid; NS, normal saline;

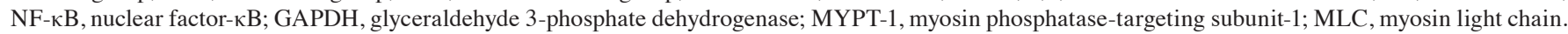

Both total and phosphorylated NF- $\mathrm{kB}$ p65 increased in the group 2, and were downregulated after Y-27632 exposure in group 3 (Fig. 4B and E).

ROCK1 and/or ROCK2 blockage has no influence on TJs in Caco-2 cells activated by TNF- $\alpha$. Following $24 \mathrm{~h}$ of stimulation with TNF- $\alpha$, ROCK1 and ROCK2 in Caco-2 cells were significantly upregulated both in mRNA and protein levels. ROCK1 and/or ROCK 2 interference blocked the corresponding expression, which was confirmed by RT-qPCR and western blotting (Fig. 5A, C and D). Then, the authors explored the contribution of each ROCK isoform toward TNF- $\alpha$-mediated activation of occludin and ZO-1. As presented in Fig. 5C and E, the expressions of occludin and ZO-1 were similar regardless of the ROCK RNAi interference and TNF- $\alpha$ treatment. RT-qPCR showed that the gene expressions of occludin and ZO-1 were in accordance with the results of western blot analysis (Fig. 5B).

ROCK1 and ROCK2 RNAi inhibit different downstream molecules in TNF- $\alpha$ treated Caco- 2 cells. TNF- $\alpha$ treatment caused an increase of phosphorylation of MYPT-1. ROCK1 blockage suppressed the phosphorylation of MYPT-1, whereas merely ROCK2 blockage had no obvious inhibition to phosphorylation of MYPT-1 (Fig. 6A and B). The phosphorylation of MLC was upregulated following TNF- $\alpha$ treatment, and it seemed that blockage of ROCK1 could inhibit MLC phosphorylation, while ROCK2 blockage only had a slight inhibition effect on phosphorylation of MLC (Fig. 6A and B).

Following this, the authors focused on the NF- $\mathrm{KB}$ signaling pathway. TNF- $\alpha$ exposure also stimulated the expressions

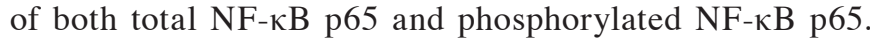
The blockage of either of the two ROCK isoforms had no impact on the suppression of the expression of total NF- $\mathrm{KB}$ p65. Knockdown of ROCK1 had no impact on TNF- $\alpha$ mediated NF- $\mathrm{BB}$ p 65 phosphorylation, whereas ROCK2 knockdown had an inhibitive impact on NF- $\mathrm{BB}$ p65 phosphorylation (Fig. 6A and C).

\section{Discussion}

An intact monolayer of intestinal epithelial cells protects the body from pathogens and other toxic luminal substances. To keep the IEB intact, it is important for prevention and treatment 

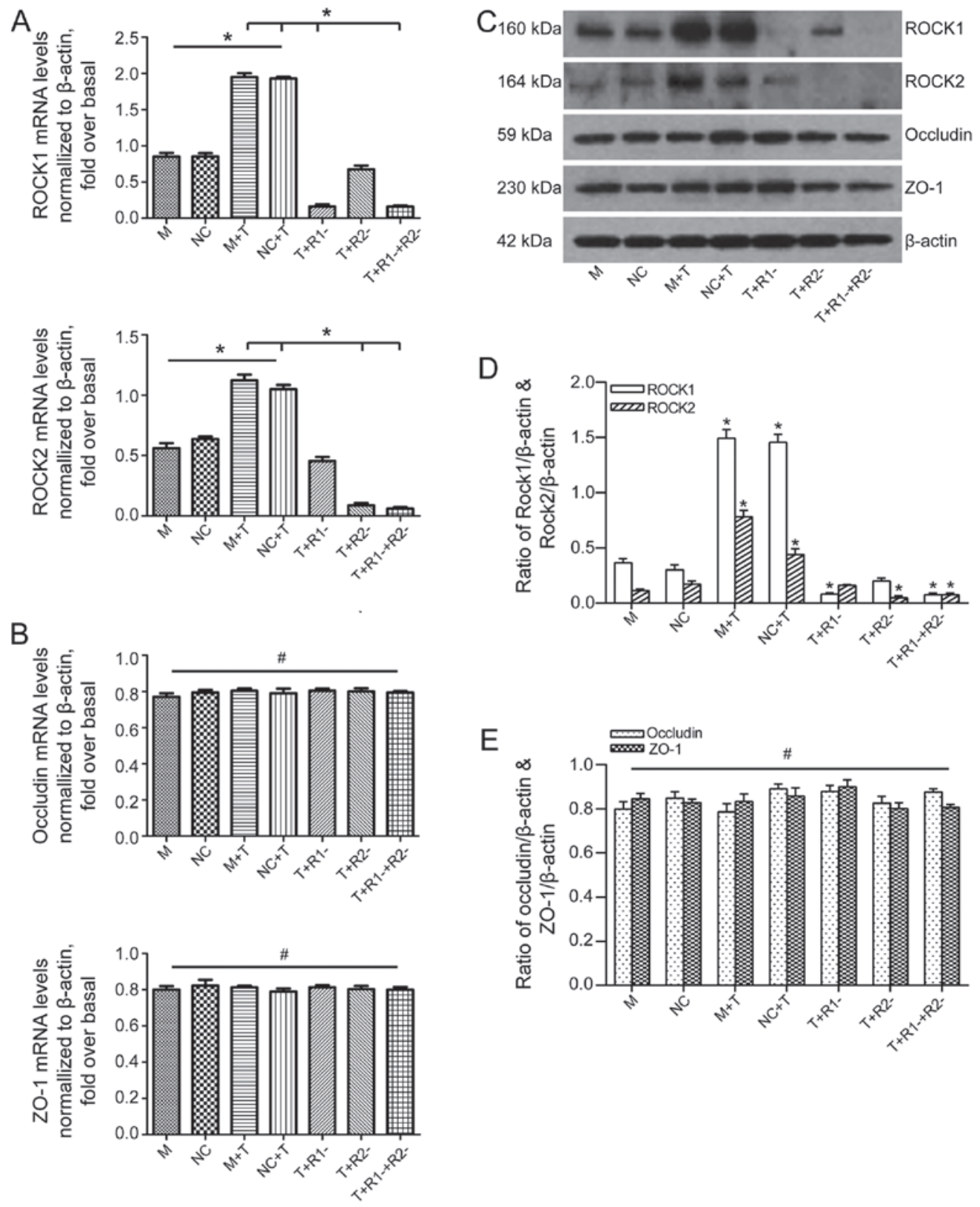

Figure 5. The effect of ROCK1 and/or ROCK2 blockage on the expression of occludin and ZO-1 after TNF- $\alpha$ activation in Caco-2 cells. (A) The levels of ROCK1 and ROCK2 mRNA expression determined by RT-qPCR. ROCK1 and ROCK2 levels were normalized with $\beta$-actin expression in each sample. (B) The levels of occludin and ZO-1 mRNA expression determined by RT-qPCR. Occludin and ZO-1 levels were normalized with $\beta$-actin expression in each sample. (C) Western blotting for ROCK1, ROCK2, occludin and ZO-1 protein levels showed as typical patterns. (D) Bars representing the relative protein quantification of ROCK1 and ROCK2 on the basis of $\beta$-actin. (E) Bars representing the relative protein quantification of occludin and ZO-1 on the basis of $\beta$-actin. Values are expressed as means \pm standard error of the mean. ${ }^{*} \mathrm{P}<0.05$ as indicated; ${ }^{*} \mathrm{P}>0.05$ as indicated. $\mathrm{M}$, mock; NC, negative control, treated with blank vehicles; M+T, mock+TNF- $\alpha$; NC+T, negative control+TNF- $\alpha$; T+R1', TNF- $\alpha+$ ROCK1 RNAi transfection; T+R2-, TNF- $\alpha+$ ROCK2 RNAi transfection; T+R1-R2- TNF- $\alpha+$ ROCK1 RNAi and ROCK2 RNAi transfection. ROCK, Rho kinase; ZO-1, zonula occludens-1; TNF- $\alpha$, tumor necrosis factor- $\alpha$; RT-qPCR, reverse transcription-quantitative polymerase chain reaction; RNAi, RNA interference.

of IBD (26). The TJs are important components of IEB, which are composed of multiple proteins including trans-membrane proteins and intracellular proteins, such as occludin and ZO-1 (27). RhoA is a member of small GTPases that is involved in numerous cellular functions, such as regulation of actin filament reorganization and cell shape. ROCK, as a downstream effector of RhoA, is an important regulator of cytoskeleton. Several studies have shown that ROCK participated in the process of intestinal inflammation and epithelial barrier dysfunction (28-30). However, the effect of ROCK in the intestinal inflammation and IEB dysfunction remains unknown.
In the present study, the authors proved that the intestinal permeability increased in TNBS-induced colitis, and ROCK inhibitor Y-27632 could alleviate the epithelial leakage. Although no difference was observed in the TJ shapes by TEM, there was a wider gap between the intestinal villa cells, tracer extravasation in colitis mice, while Y-27632 reversed these effects. That meant that the ROCK inhibitor could improve intestinal permeability through improving and enhancing TJ function, not shape.

Furthermore, the authors evaluated the expression levels of intestinal TJs with or without ROCK inhibitor. The decrease 

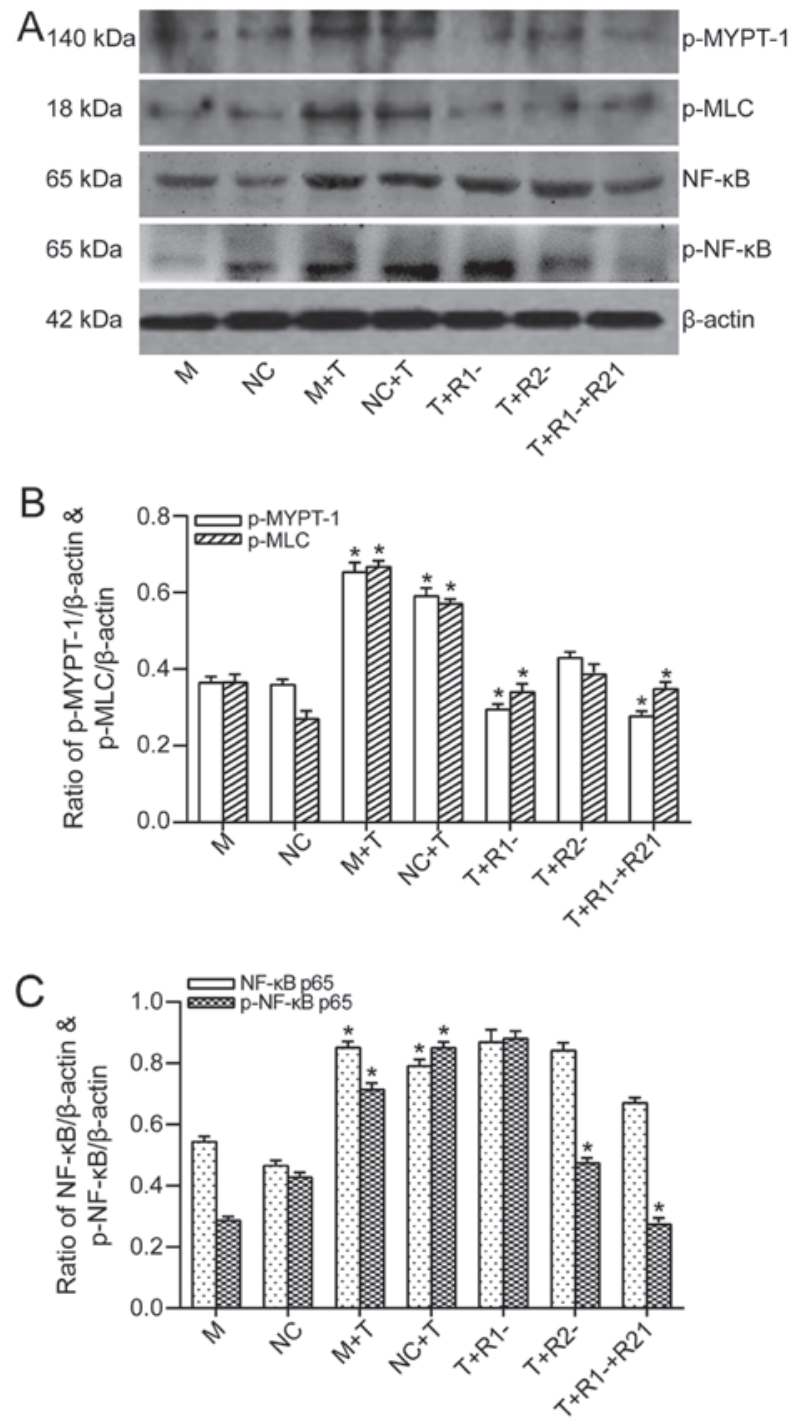

Figure 6. The effect of ROCK1 and/or ROCK2 blockage on the expression of downstream molecular of ROCK signal pathway after TNF- $\alpha$ activation in Caco-2 cells. (A) Western blot analysis for p-MYPT-1, p-MLC, NF-кB p65 and $\mathrm{p}-\mathrm{NF}-\mathrm{kB}$ p 65 protein levels showed as typical patterns. (B) Bars representing the relative protein quantification of p-MYPT-1 and p-MLC on the basis of $\beta$-actin. ${ }^{~} \mathrm{P}<0.05$ for $\mathrm{T}$ group vs. $\mathrm{M}$ or $\mathrm{NC}$ group, $\mathrm{T}+\mathrm{R} 1{ }^{-}$group vs. $\mathrm{T}$ group, and $\mathrm{T}+\mathrm{R} 1^{-}+\mathrm{R} 2^{-}$groups vs. $\mathrm{T}$ group. (C) Bars representing the relative protein quantification of NF-kB p65 and p-NF- $\mathrm{kB}$ p 65 on the basis of $\beta$-actin. ${ }^{*} \mathrm{P}<0.05$ for $\mathrm{T}$ group vs. $\mathrm{M}$ or $\mathrm{NC}$ group, $\mathrm{T}+\mathrm{R} 2^{-}$group vs. $\mathrm{T}$ group, and $\mathrm{T}+\mathrm{R} 1^{-}+\mathrm{R} 2$ groups vs. T group. Values are expressed as means \pm standard error of the mean. $\mathrm{M}$, mock; $\mathrm{NC}$, negative control, treated with blank vehicles; $\mathrm{M}+\mathrm{T}$, mock+TNF- $\alpha$; NC+T, negative control+TNF- $\alpha ; \mathrm{T}+\mathrm{R} 1$; TNF- $\alpha+\mathrm{ROCK} 1$ RNAi transfection; $\mathrm{T}+\mathrm{R} 2-\mathrm{-}$, TNF- $\alpha+\mathrm{ROCK} 2$ RNAi transfection; $\mathrm{T}+\mathrm{R} 1^{-}+\mathrm{R} 2$, TNF- $\alpha+$ ROCK1 RNAi and ROCK2 RNAi transfection. ROCK, Rho kinase; NF- $\kappa \mathrm{B}$, nuclear factor- $\kappa \mathrm{B}$; RNAi, RNA interference; MYPT-1, myosin phosphatase-targeting subunit-1; MLC, myosin light chain.

of occludin and ZO-1 in TNBS colitis mice is accordance with the results of Ji et al (31). In addition, the authors confirmed ROCK inhibitor could upregulate the two substances expression. It is reported that Y-27623 could ameliorate ethanol induced $\mathrm{ZO}-1$ decrease and redistribution (32). The results furthermore confirmed Y-27632 changed the ZO-1 expression both in mRNA and in protein levels. Occludin was thought of one of the downstream molecules of ROCK and could be phosphorylated by ROCK activation. However, there was no report hitherto that ROCK could increase the expressions of either the gene or protein of occludin (33). The upregulation of occludin mRNA and protein levels in the present study was probably due to the multiple interference factors in systemic inflammation.

In addition, the authors also demonstrated that two ROCK signal pathways were activated during TNBS-induced colitis, including the MLC pathway and the NF- $\kappa B$ pathway. There was an upregulation of phosphorylation of MLC but not MYPT-1, which indicated that ROCK may affect the cytoskeleton structure via activating MLC. ROCK inhibitor caused a significant decrease of both p-MYPT-1 and p-MLC, suggesting that besides inhibiting the phosphorylation of MLC directly, Y-27632 could activate MLCP through suppressing MYPT-1 activation, thus inhibiting MLC indirectly. Since MLC is one of the most important proteins of the cytoskeleton structure, the authors believe that ROCK inhibitor improving the TJ function via inhibiting MLC activation may be one of the mechanisms.

The NF- $\mathrm{kB}$ signaling pathway is also activated in TNBSinduced colitis, which is in accordance with Segain et al (20). The phosphorylation at the site Ser536 detected by western blotting confirmed the NF- $\kappa \mathrm{B}$ activation. As NF- $\kappa \mathrm{B}$ is one of the key factors regulating the transcription of multiple inflammatory molecules, the authors speculated that ROCK activation may promote the generation of inflammatory molecules through activating NF- $\kappa B$ and thus lead to an acceleration of intestinal epithelial damage.

In Caco-2 cells, it turned out that there was no difference of occludin and ZO-1 expressions between TNF- $\alpha$ and ROCK RNAi treated groups. That confirmed the speculation that there was no direct relationship between the expression of occludin/ZO-1 and ROCK, with or without TNF- $\alpha$ treatment. The variation of TJs levels may ascribe to the activation of multiple signaling pathways and the production of large amount of inflammatory factors.

The authors further evaluated the different roles of ROCK1 and ROCK2 in recognizing the downstream molecules in Caco- 2 cell lines separately. Following TNF- $\alpha$ treatment, the dual ROCK1/ROCK2 blockage significantly inhibited phosphorylation of the ROCK substrates such as MLC, MYPT-1 and NF- $\kappa$ B. However, individual knockdown of ROCK1 could significantly suppress phosphorylation of MYPT-1 and MLC. While sole ROCK 2 knockdown only had a slight inhibitory effect on phosphorylation of MYPT-1 and MLC. That meant the activation of MYPT-1 an MLC depended more on ROCK1 than on ROCK2.

The authors then turned their attention to the NF- $\mathrm{KB}$ pathway. The total expression of NF- $\mathrm{KB}$ was upregulated following TNF- $\alpha$ treatment, while the inhibition of ROCK1 and/or ROCK2 did not alter the expression of NF-кB. However, the phosphorylation of NF- $\mathrm{KB}$ was inhibited more significantly when ROCK2 were blocked, suggesting that ROCK2 is more important in the activation of the NF- $\mathrm{KB}$ pathway. The results are in accordance to the studies of Shimada and Rajagopalan (34) using a human endothelial cell line HUVECs. In the present study, we did not completely clarify the exact action of the two ROCK isoform differences in TNF- $\alpha$-treated Caco- 2 cells. More complex signal transduction pathways may be involved in the pathogenesis of intestinal inflammation.

In conclusion, the present study confirmed that ROCK inhibitor could alleviate colitis and IEB dysfunction; ROCK 
downstream signaling pathways (MLC and NF- $\mathrm{B}$ ) were activated in colitis mice and inhibited by Y-27632, which improved IEB dysfunction. ROCK1 and ROCK2 knockout separately played different roles in recognizing the downstream molecules. Thus, ROCK inhibitor may be a potential therapeutic method in IBD and further studies are required to further clarify the exact mechanism of ROCK and its pathway in regulating $\mathrm{TJ}$ function in IEB.

\section{Acknowledgements}

The present study was supported by the National Natural Science Foundation of China (grant nos. 81400628 and 81500460) and the Science and Technology Commission of Shanghai Municipality (grant no. 16411952300) and the Shanghai Municipal Health Bureau (grant no. 201440392).

\section{References}

1. Zeissig S, Bojarski C, Buergel N, Mankertz J, Zeitz M, Fromm M and Schulzke JD: Downregulation of epithelial apoptosis and barrier repair in active Crohn's disease by tumour necrosis factor alpha antibody treatment. Gut 53: 1295-1302, 2004.

2. Turck D, Ythier H, Maquet E, Deveaux M, Marchandise X, Farriaux JP and Fontaine G: Intestinal permeability to [51Cr] EDTA in children with Crohn's disease and celiac disease. J Pediatr Gastroenterol Nutr 6: 535-537, 1987.

3. May GR, Sutherland LR and Meddings JB: Is small intestinal permeability really increased in relatives of patients with Crohn's disease? Gastroenterology 104: 1627-1632, 1993.

4. Clayburgh DR, Shen L and Turner JR: A porous defense: The leaky epithelial barrier in intestinal disease. Lab Invest 84: 282-291, 2004

5. Katz KD, Hollander D, Vadheim CM, McElree C, Delahunty T, Dadufalza VD, Krugliak P and Rotter JI: Intestinal permeability in patients with Crohn's disease and their healthy relatives. Gastroenterology 97: 927-931, 1989.

6. Edelblum KL and Turner JR: The tight junction in inflammatory disease: Communication breakdown. Curr Opin Pharmacol 9: 715-720, 2009

7. Shen L, Weber CR and Turner JR: The tight junction protein complex undergoes rapid and continuous molecular remodeling at steady state. J Cell Biol 181: 683-695, 2008.

8. Hering NA and Schulzke JD: Therapeutic options to modulate barrier defects in inflammatory bowel disease. Dig Dis 27: 450-454, 2009.

9. Steed E, Balda MS and Matter K: Dynamics and functions of tight junctions. Trends Cell Biol 20: 142-149, 2010.

10. Ivanov AI: Structure and regulation of intestinal epithelial tight junctions: Current concepts and unanswered questions. Adv Exp Med Biol 763: 132-148, 2012.

11. Sebbagh M, Renvoizé C, Hamelin J, Riché N, Bertoglio J and Bréard J: Caspase-3-mediated cleavage of ROCK I induces MLC phosphorylation and apoptotic membrane blebbing. Nat Cell Biol 3: 346-352, 2001

12. Chaturvedi LS, Marsh HM and Basson MD: Role of RhoA and its effectors ROCK and mDia1 in the modulation of deformationinduced FAK, ERK, p38, and MLC motogenic signals in human Caco-2 intestinal epithelial cells. Am J Physiol Cell Physiol 301: C1224-C1238, 2011.

13. Sapet C, Simoncini S, Loriod B, Puthier D, Sampol J, Nguyen C, Dignat-George $\mathrm{F}$ and Anfosso F: Thrombin-induced endothelial microparticle generation: Identification of a novel pathway involving ROCK-II activation by caspase-2. Blood 108: 1868-1876, 2006.

14. Turner MS, Fen-Fen-Lin, Trauger JW, Stephens J and LoGrasso P: Characterization and purification of truncated human Rho-kinase II expressed in Sf-21 cells. Arch Biochem Biophys 405: 13-20, 2002.

15. Chen XQ, Tan I, Ng CH, Hall C, Lim L and Leung T: Characterization of RhoA-binding kinase ROKalpha implication of the pleckstrin homology domain in ROKalpha function using region-specific antibodies. J Biol Chem 277: 12680-12688, 2002.
16. Matsui T, Amano M, Yamamoto T, Chihara K, Nakafuku M, Ito M, Nakano T, Okawa K, Iwamatsu A and Kaibuchi K: Rho-associated kinase, a novel serine/threonine kinase, as a putative target for small GTP binding protein Rho. EMBO J 15: 2208-2216, 1996

17. Ward Y, Yap SF, Ravichandran V, Matsumura F, Ito M, Spinelli B and Kelly K: The GTP binding proteins Gem and Rad are negative regulators of the Rho-Rho kinase pathway. J Cell Biol 157: 291-302, 2002

18. Leung T, Chen XQ, Manser E and Lim L: The p160 RhoA-binding kinase ROK alpha is a member of a kinase family and is involved in the reorganization of the cytoskeleton. Mol Cell Biol 16: 5313-5327, 1996.

19. Ivanov AI, Parkos CA and Nusrat A: Cytoskeletal regulation of epithelial barrier function during inflammation. Am J Pathol 177: 512-524, 2010.

20. Segain JP, Raingeard de la Blétière D, Sauzeau V, Bourreille A, Hilaret G, Cario-Toumaniantz C, Pacaud P, Galmiche JP and Loirand G: Rho kinase blockade prevents inflammation via nuclear factor kappa B inhibition: Evidence in Crohn's disease and experimental colitis. Gastroenterology 124: 1180-1187, 2003.

21. Mihaescu A, Santén S, Jeppsson B and Thorlacius H: Rho kinase signalling mediates radiation-induced inflammation and intestinal barrier dysfunction. Br J Surg 98: 124-131, 2011.

22. AnwarKN,FazalF,MalikAB andRahman A:RhoA/Rho-associated kinase pathway selectively regulates thrombin-induced intercellular adhesion molecule-1 expression in endothelial cells via activation of I kappa B kinase beta and phosphorylation of RelA/p65. J Immunol 173: 6965-6972, 2004

23. Rodriguez PL, Sahay S, Olabisi OO and Whitehead IP: ROCK I-mediated activation of NF-kappaB by RhoB. Cell Signal 19: 2361-2369, 2007.

24. Voiosu T, Benguş A, Dinu R, Voiosu AM, Bălănescu P, Băicuş C, Diculescu M, Voiosu R and Mateescu B: Rapid fecal calprotectin level assessment and the SIBDQ score can accurately detect active mucosal inflammation in IBD patients in clinical remission: A prospective study. J Gastrointestin Liver Dis 23: 273-278, 2014.

25. Ameho CK, Adjei AA, Harrison EK, Takeshita K, Morioka T, Arakaki Y, Ito E, Suzuki I, Kulkarni AD, Kawajiri A, et al: Prophylactic effect of dietary glutamine supplementation on interleukin- 8 and tumour necrosis factor alpha production in trinitrobenzene sulphonic acid induced colitis. Gut 41: 487-493, 1997.

26. Dong X, Liu Z, Lan D, Niu J, Miao J, Yang G, Zhang F, Sun Y, Wang $\mathrm{K}$ and Miao Y: Critical role of Keratin 1 in maintaining epithelial barrier and correlation of its down-regulation with the progression of inflammatory bowel disease. Gene 608: 13-19, 2017.

27. Turner JR, Buschmann MM, Romero-Calvo I, Sailer A and Shen L: The role of molecular remodeling in differential regulation of tight junction permeability. Semin Cell Dev Biol 36: 204-212, 2014.

28. Moyer RA, Wendt MK, Johanesen PA, Turner JR and Dwinell MB: Rho activation regulates CXCL12 chemokine stimulated actin rearrangement and restitution in model intestinal epithelia. Lab Invest 87: 807-817, 2007.

29. Le Dréan G, Haure-Mirande V, Ferrier L, Bonnet C, Hulin P, de Coppet $P$ and Segain JP: Visceral adipose tissue and leptin increase colonic epithelial tight junction permeability via a RhoA-ROCK-dependent pathway. FASEB J 28: 1059-1070, 2014.

30. Elamin E, Masclee A, Dekker J and Jonkers D: Ethanol disrupts intestinal epithelial tight junction integrity through intracellular calcium-mediated Rho/ROCK activation. Am J Physiol Gastrointest Liver Physiol 306: G677-G685, 2014.

31. Ji R, Wang A, Shang H, Chen L, Bao C, Wu L, Wu H and Shi Y: Herb-partitioned moxibustion upregulated the expression of colonic epithelial tight junction-related proteins in Crohn's disease model rats. Chin Med 11: 20, 2016.

32. Tong J, Wang Y, Chang B, Zhang D and Wang B: Y-27632 inhibits ethanol-induced increase in intestinal epithelial barrier permeability. Mol Med Rep 9: 2357-2361, 2014.

33. Furuse M, Hirase T, Itoh M, Nagafuchi A, Yonemura S, Tsukita $S$ and Tsukita S: Occludin: A novel integral membrane protein localizing at tight junctions. J Cell Biol 123: 1777-1788, 1993.

34. Shimada $\mathrm{H}$ and Rajagopalan LE: Rho kinase-2 activation in human endothelial cells drives lysophosphatidic acid-mediated expression of cell adhesion molecules via NF-kappaB p65. J Biol Chem 285: 12536-12542, 2010. 